\title{
Cross-reactivity of human monoclonal antibodies generated with peripheral blood lymphocytes from dengue patients with Japanese encephalitis virus
}

\author{
Chonlatip Pipattanaboon 1,3,8,* \\ Tadahiro Sasaki ${ }^{2,8, *}$ \\ Mitsuhiro Nishimura ${ }^{2,8}$ \\ Chayanee Setthapramote ${ }^{1,8}$ \\ Pannamthip Pitaksajjakul ${ }^{1,4,8}$ \\ Pornsawan Leaungwutiwong ${ }^{1,3,8}$ \\ Kriengsak Limkittikul ${ }^{5,8}$ \\ Orapim Puiprom ${ }^{6}$ \\ Mikiko Sasayama ${ }^{6}$ \\ Panjaporn Chaichana ${ }^{6}$ \\ Tamaki Okabayashi ${ }^{6}$ \\ Takeshi Kurosu ${ }^{2,8}$ \\ Ken-ichiro Ono 7,8 \\ Pongrama Ramasoota ${ }^{1,4,8}$ \\ Kazuyoshi Ikuta ${ }^{2,8}$ \\ 'Center of Excellence for Antibody Research, \\ Faculty of Tropical Medicine, Mahidol \\ University, Bangkok, Thailand; ${ }^{2}$ Department \\ of Virology, Research Institute for Microbial \\ Diseases, Osaka University, Osaka, Japan; \\ ${ }^{3}$ Department of Microbiology and Immunology, \\ ${ }^{4}$ Department of Social and Environmental \\ Medicine, ${ }^{5}$ Department of Tropical Pediatrics, \\ Faculty of Tropical Medicine, Mahidol \\ University, ${ }^{6}$ Mahidol-Osaka Center for \\ Infectious Diseases, Bangkok, Thailand; ${ }^{7}$ Medical \\ and Biological Laboratories Corporation \\ Ltd, Nagano, Japan; ${ }^{8}$ JST/JICA, Science \\ and Technology Research Partnership for \\ Sustainable Development, Tokyo, Japan \\ "These authors made an equal contribution \\ to this study
}

Correspondence: Pongrama Ramasoota Department of Social and Environmental Medicine, Faculty of Tropical Medicine, Mahidol University, Ratchathewi,

Bangkok, Thailand

Tel +66 23549100

Fax +6626435616

Email pongrama.ram@mahidol.ac.th

Kazuyoshi lkuta

Department of Virology, Research Institute

for Microbial Diseases, Osaka University, Suita,

Osaka 565-087I, Japan

Tel +81668798307

Fax +81668798310

Email ikuta@biken.osaka-u.ac.jp
This article was published in the following Dove Press journal:

Biologics: Targets and Therapy

14 August 2013

Number of times this article has been viewed

Background: Hybridomas that produce human monoclonal antibodies (HuMAbs) against Dengue virus (DV) had been prepared previously using peripheral blood lymphocytes from patients with DV during the acute and convalescent phases of a secondary infection. Anti-DV envelope glycoprotein (E) 99 clones, anti-DV premembrane protein (prM) 8 clones, and antiDV nonstructural protein 1 (NS1) 4 clones were derived from four acute-phase patients, and anti-DV E 2 clones, anti-DV prM 2 clones, and anti-DV NS1 8 clones were derived from five convalescent-phase patients.

Methods and results: In the present study, we examined whether these clones cross-reacted with Japanese encephalitis virus (JEV), which belongs to the same virus family. Forty-six of the above-described 99 (46/99) anti-E, 0/8 anti-prM, and 2/4 anti-NS1 HuMAbs from acutephase, and 0/2 anti-E, 0/2 anti-prM, and 5/8 anti-NS1 HuMAbs from convalescent-phase showed neutralizing activity against JEV. Thus, most of the anti-E and anti-NS1 (but not the anti-prM) antibodies cross-reacted with JEV and neutralized this virus. Interestingly, 3/46 anti-E HuMAbs derived from acute-phase patients and 3/5 anti-NS1 HuMAbs from convalescent-phase patients showed particularly high neutralizing activity against JEV. Consequently, the HuMAbs showing neutralization against JEV mostly consisted of two populations: one was HuMAbs recognizing DV E and showing neutralization activity against all four DV serotypes (complex-type) and the other was HuMAbs recognizing DV NS1 and showing subcomplex-type cross-reaction with DV.

Conclusion: Anti-DV E from acute phase (46/99) and anti-DV NS1 (7/12) indicate neutralizing activity against JEV. In particular, three of 46 anti-DV E clones from acute phase and three of five anti-NS1 clones from convalescent phase showed strong neutralizing activity against JEV.

Keywords: Dengue virus, Japanese encephalitis virus, viral neutralization, human monoclonal antibody, envelope, nonstructural protein 1

\section{Introduction}

Dengue virus (DV) encodes capsid protein (C), premembrane protein (prM), and envelope glycoprotein (E), in addition to seven nonstructural proteins (NS). ${ }^{1}$ There are four antigenically distinct serotypes (DV1-DV4), which share major antigens with each other and with other mosquito-borne and tick-borne flaviviruses, including Japanese encephalitis virus (JEV). ${ }^{2-8} \mathrm{DV}$ and JEV are closely related, belonging to the same virus family, Flaviviridae. Both viruses are cocirculating in areas of Southeast Asia, including Thailand. ${ }^{9}$ Indeed, vaccination rates against JEV in Thailand are high, at $84 \%$ in 1998 and $98 \%$ in $2008 .^{5}$

The immune response to a primary DV infection generates anti-DV neutralizing antibodies, which then protect against subsequent infection by the same serotype. ${ }^{10}$ 
However, severe dengue infections often occur in patients who are secondarily infected with a different DV serotype. ${ }^{10}$ The reason for this may be that the second virus uses preexisting anti-DV antibodies (raised during the primary infection) to gain entry to macrophages expressing Fc receptors, a process called antibody-dependent enhancement. ${ }^{11,12}$ Interestingly, most DV infections are asymptomatic, ${ }^{13}$ even in individuals who are secondarily infected with a heterotypic DV. ${ }^{14}$ However, in symptomatic cases, it can cause a wide spectrum, ranging from a mild illness, such as dengue fever, to severe illnesses, such as dengue hemorrhagic fever and dengue shock syndrome. ${ }^{15}$

There have been several trials examining the clinical implications of prior exposure to JEV, or vaccination against JEV, which may increase the severity of subsequent DV infections. The results showed that neutralizing antibodies against JEV have both protective and detrimental effects upon subsequent DV infection. ${ }^{8,16-20}$

Examination of the humoral immune status of DVinfected individuals, including dengue patients in the acute and convalescent phases of the secondary infection with heterotypic DV, may provide valuable information that will inform the development of anti-dengue vaccines. Previous reports showed that antibodies raised during primary infections were more type-specific, whereas those raised during secondary infections were more heterogeneous and wideranging in their ability to cross-react with heterotypes. ${ }^{21,22}$ Several groups have reported successful generation of hybridomas that produce anti-DV human monoclonal antibodies (HuMAbs), ${ }^{22-25}$ and all used peripheral blood mononuclear cells isolated from patients during the convalescent phases of primary and secondary infections. However, there are no reports of hybridomas being generated using peripheral blood mononuclear cells derived from the acute phase of a secondary DV infection. Information on the anti-DV antibodies derived from patients during the acute phase after secondary infection could be useful for understanding the mechanism(s) underlying dengue immunopathogenicity.

Recently, we reported the preparation of several hybridomas that secrete anti-DV HuMAbs by using peripheral blood mononuclear cells from dengue patients at the acute and convalescent phases of secondary infection with DV. ${ }^{26,27}$

The aim of the present study was to investigate whether these dengue patient-derived HuMAbs showed neutralizing activity against JEV. The results showed that two populations of HuMAbs, anti-E from acute-phase patients and anti-NS1 from convalescent-phase patients, showed neutralizing activity against JEV at high rates.

\section{Materials and methods Cell lines and viruses}

Previously, 121 hybridomas were derived from dengue patients during the acute phase of a secondary DV infection and 15 were derived from patients during the convalescent phase. ${ }^{26}$ For the present study, Vero cells were cultured in minimum essential medium supplemented with $10 \%$ fetal bovine serum and maintained in a $5 \% \mathrm{CO}_{2}$ incubator at $37^{\circ} \mathrm{C}$. The mosquito-derived cell line, $\mathrm{C} 6 / 36$, was cultured at $28^{\circ} \mathrm{C}$ in Leibovitz's L-15 medium supplemented with $10 \%$ minimum essential medium and $0.3 \%$ tryptose phosphate broth. JEV (Nakayama strain) was cultured in C6/36 cells and the culture supernatants were used as viral stocks. Infectivity titers were estimated in Vero cells according to the number of focus-forming units, as previously described. ${ }^{28}$

\section{Immunofluorescence assay}

Vero cells were plated in 96-well plates at a density of $2.5 \times 10^{4}$ per well and either mock-infected or infected with JEV. After incubation for 16 hours, the cells were fixed with 3.7\% formaldehyde in phosphate-buffered saline and permeabilized with $1 \%$ Triton X-100 in phosphatebuffered saline. Undiluted hybridoma culture fluid was used as a source of HuMAbs. Bound antibodies were visualized using Alexa Fluor 488-conjugated anti-human or anti-mouse IgG secondary antibody (1:1,000, Invitrogen, Carlsbad, CA, USA).

\section{Viral neutralization assay}

The viral neutralization assay was performed using undiluted hybridoma culture fluid as previously described ${ }^{29}$ The fluid, or Dulbecco's Modified Eagle's Medium with 15\% fetal bovine serum (as a negative control), was mixed with 100 focus-forming units of JEV $(25 \mu \mathrm{L})$. After incubating for one hour, the mixture was used to infect Vero cells in a 96-well microplate. After inoculation at $37^{\circ} \mathrm{C}$ for 2 hours, $100 \mu \mathrm{L}$ of minimum essential medium containing $3 \%$ fetal bovine serum was added to the wells. After a further overnight incubation at $37^{\circ} \mathrm{C}$, the cells were fixed with $3.7 \%$ formaldehyde in phosphate-buffered saline and permeabilized with $1 \%$ Triton X-100 in phosphate-buffered saline. The plates were then stained with murine anti-E 4G2 monoclonal antibody ${ }^{30}$ at $4{ }^{\circ} \mathrm{C}$ overnight for the immunofluorescence assay, as above. Bound antibodies were visualized using an Alexa Fluor 488-conjugated anti-mouse IgG (1:1,000; Invitrogen). All assays were performed in duplicate and the results were expressed as the mean. The viral neutralization 
activity of HuMAbs generated by the hybridoma clones was expressed as follows:,$-<50 \% ;+, 50 \%$ to $<80 \%$; or ,$++ \geq 80 \%$ reduction of focus-forming units compared with the negative control.

\section{Western blot analysis}

The JEV-infected Vero cells were suspended in loading buffer containing 2-mercaptoethanol, electrophoresed in 10\% sodium dodecyl sulfate polyacrylamide gels, then blotted onto polyvinylidene fluoride membranes. The blots were then incubated with undiluted hybridoma culture fluid at $4{ }^{\circ} \mathrm{C}$ overnight, followed by incubation with horseradish peroxidase-conjugated anti-human IgG (Jackson ImmunoResearch Laboratories, West Grove, PA, USA) for a further 2 hours at room temperature. The peroxidase reaction was visualized using ECL Plus Western Blotting Detection System (GE Healthcare UK Ltd, Little Chalfont, UK).

\section{Results}

\section{Patient demographics}

In total, peripheral blood mononuclear cell samples from nine secondarily infected dengue patients were used for anti-DV HuMAbs. ${ }^{26}$ The peripheral blood mononuclear cells from D23, D32, and D33 (who had dengue fever), and D30 (who had dengue hemorrhagic fever) were obtained 6-8 days after the onset of fever (acute phase), and those from D25, D26, and D28 (who had dengue fever), and
D22 and D27 (who had dengue hemorrhagic fever) were obtained between 12 and 15 days after the onset of fever (convalescent phase). The four acute-phase patients were all infected with DV2.

\section{Cross-reactivity of HuMAbs with JEV}

A previous study examined the serologic activity of the HuMAbs against all four serotypes using immunofluorescence and viral neutralization assays. ${ }^{26}$ The present study went on to examine possible cross-reactions with JEV-infected cells by immunofluorescence. The results are summarized in Table 1. The results from a DV2-derived viral protein expression system showed that 99, eight, and four of the $121 \mathrm{HuMAbs}$ derived from four acute-phase patients recognized DV E, prM, and NS1, respectively, whereas two, two, and eight of $15 \mathrm{HuMAbs}$ derived from five convalescent-phase patients recognized DV E, prM, and NS1, respectively. ${ }^{26}$ As summarized in Table 2, immunofluorescence analysis showed that 93 of the above-described 99 (93/99) anti-E clones, 1/8 antiprM clones, and 1/4 anti-NS1 clones (anti-NS1 D30-2B1G5 also showed a weak reaction with $\mathrm{E}$ ) derived from acutephase patients were cross-reactive with JEV, whereas $2 / 2$ anti-E clones and 0/8 anti-prM clones were cross-reactive with JEV, and 1/8 anti-NS1 clones were cross-reactive with JEV (anti-NS1 D25-2B11G11 also showed a weak reaction with E and prM, and anti-NS1 D25-4D4F10 also reacted weakly with prM).

Table I Patient disease status and the human monoclonal antibodies obtained in this study

\begin{tabular}{|c|c|c|c|c|c|c|c|c|}
\hline \multirow{3}{*}{$\begin{array}{l}\text { Dengue patient's clinical } \\
\text { phase for HuMAb } \\
\text { preparation }\end{array}$} & \multirow[t]{3}{*}{$\begin{array}{l}\text { Reaction with } \\
\text { DV2 protein }\end{array}$} & \multirow[t]{3}{*}{$\begin{array}{l}\text { Total HuMAb } \\
\text { clone number }\end{array}$} & \multirow{3}{*}{$\begin{array}{l}\text { Patient } \\
\text { (clone } \\
\text { number) }\end{array}$} & \multicolumn{5}{|c|}{$\begin{array}{l}\text { Dengue patient-derived HuMAb } \\
\text { clone number }\end{array}$} \\
\hline & & & & \multicolumn{2}{|c|}{ IF with JEV } & \multicolumn{3}{|c|}{ VN against JEV ${ }^{a}$} \\
\hline & & & & - & + & - & + & ++ \\
\hline \multirow[t]{7}{*}{ Acute } & E & 99 & D23 (56) & 5 & 51 & 32 & 23 & 1 \\
\hline & & & D30 (23) & 0 & 23 & 10 & 12 & I \\
\hline & & & D32 (5) & I & 4 & 2 & 2 & 1 \\
\hline & & & D33 (I5) & 0 & 15 & 9 & 6 & 0 \\
\hline & $\mathrm{prM}$ & 8 & D23 (8) & 8 & 0 & 8 & 0 & 0 \\
\hline & NSI & 4 & D23 (3) & 3 & 0 & 2 & 1 & 0 \\
\hline & & & D30 (I) & 0 & $\mathrm{I}^{\mathrm{b}}$ & 0 & $\mathrm{I}^{\mathrm{b}}$ & 0 \\
\hline \multirow[t]{8}{*}{ Convalescent } & E & 2 & D22 (I) & 0 & 1 & I & 0 & 0 \\
\hline & & & D26 (I) & 0 & I & I & 0 & 0 \\
\hline & $\mathrm{prM}$ & 2 & D22 (I) & I & 0 & I & 0 & 0 \\
\hline & & & D27 (I) & 1 & 0 & I & 0 & 0 \\
\hline & NSI & 8 & D25 (5) & $4^{\mathrm{c}, \mathrm{d}}$ & 1 & $2^{c}$ & $2^{\mathrm{d}}$ & 1 \\
\hline & & & D26 (I) & 1 & 0 & 0 & 0 & 1 \\
\hline & & & D27 (I) & I & 0 & 0 & 0 & 1 \\
\hline & & & D28 (I) & 1 & 0 & $\mathrm{I}$ & 0 & 0 \\
\hline
\end{tabular}

Notes: ${ }^{a}-,<50 \% ;+, \geq 50 \%$ and $<80 \%$; and,$++ \geq 80 \%$ FFU reduction compared with the negative control; ${ }^{b}$ HuMAb D30-2BIG5 also shows a weak reaction with DV2 E; cHuMAb D25-4D4FIO also shows a weak reaction with DV2 prM; ${ }^{\circ}$ HuMAb D25-2BI IGII also shows a weak reaction with DV2 E and prM.

Abbreviations: DV, Dengue virus; E, envelope glycoprotein; HuMAb, human monoclonal antibody; prM, premembrane protein; NSI, nonstructural protein I; IF, immunofluorescence; VN, viral neutralization; FFU, focus-forming units; JEV, Japanese encephalitis virus. 
Table 2 Cross-reactivity of Japanese encephalitis virus with human monoclonal antibodies derived from dengue patients by immunofluorescence

\begin{tabular}{|c|c|c|c|c|c|c|c|c|c|}
\hline \multicolumn{4}{|c|}{ DV serotype } & \multicolumn{6}{|c|}{ HuMAb clones reactive with DV by IF (reactive with JEV by IF) } \\
\hline \multirow[t]{2}{*}{ DVI } & \multirow[t]{2}{*}{ DV2 } & \multirow[t]{2}{*}{ DV3 } & \multirow[t]{2}{*}{ DV4 } & \multicolumn{3}{|c|}{ Derived from acute-phase patients ${ }^{\mathrm{b}}$} & \multicolumn{3}{|c|}{ Derived from convalescent-phase patients ${ }^{b}$} \\
\hline & & & & $\mathbf{E}$ & prM & NSI & $\mathbf{E}$ & prM & NSI \\
\hline+ & + & - & - & & & & & & $\mathrm{I}(0)$ \\
\hline- & + & + & - & I $(0)^{\mathrm{a}}$ & & & & & \\
\hline+ & + & + & - & & & $3^{c}\left(I^{c}\right)$ & & & $5(0)$ \\
\hline+ & + & - & + & $2(2)$ & & & & & \\
\hline+ & + & + & + & $96(91)$ & $8(0)$ & I (0) & $2(2)$ & $2(0)$ & $2^{\mathrm{d}, \mathrm{e}}\left(I^{\mathrm{d}}\right)$ \\
\hline
\end{tabular}

Notes: ${ }^{\mathrm{T}}$ The number of HuMAb clones showing a positive reaction with JEV-infected cells by IF is shown in parentheses; bthe target viral proteins for the HuMAbs were determined using E, prM, and NSI proteins derived from DV2; 26 c HuMAb D30-2BI G5 is also weakly reactive with DV2 E; d ${ }^{\circ}$ HMAb D25-2BI IGII is also weakly reactive with DV2 E and prM; eHuMAb D25-4D4FI0 is also weakly reactive with DV2 prM.

Abbreviations: DV, Dengue virus; E, envelope glycoprotein; HuMAb, human monoclonal antibody; prM, premembrane protein; NSI, nonstructural protein I; IF, immunofluorescence; JEV, Japanese encephalitis virus.

The results of the viral neutralization assay revealed that 46/99 anti-E, 0/8 anti-prM, and 2/4 anti-NS1 derived from acute-phase showed neutralizing activity against JEV, whereas $0 / 2$ anti-E, $0 / 2$ anti-prM, and 5/8 anti-NS1 derived from convalescent-phase showed neutralizing activity against JEV (Table 3). Interestingly, three anti-E HuMAbs derived from acute-phase and three anti-NS1 HuMAbs derived from convalescent-phase showed very high viral neutralization activity against JEV, as indicated by ++ (Table 1) and clone numbers on the right side in parentheses (Table 3). There were no viral neutralization-positive clones among HuMAbs recognizing prM, irrespective of their origins (acute or convalescent phase). The characteristics of the individual HuMAbs are summarized in Table S1. Of the three clones that reacted with DV NS1 but also showed a weak cross-reaction with $\mathrm{E}$ and/or prM in the immunofluorescence with 293T cells transfected with individual DV2 viral genes, ${ }^{26}$ D30-2B1G5 and D25-2B11G11, but not D25-4D4F10, showed neutralization against JEV.

Most of the HuMAb clones showing a positive reaction with JEV in immunofluorescence recognized DV E and showed a complex-type cross-reaction with all four serotypes (91/96 clones [95\%], Table 2). Similarly, viral neutralization assay showed that 46/99 (46\%) anti-E and 2/4 (50\%) anti-NS1 HuMAbs from acute-phase showed neutralizing activity against JEV, as did 5/8 (63\%) anti-NS1 HuMAbs from convalescent-phase (Table 3). Interestingly, 38/46 of the above anti-E (83\%) were HuMAbs showing neutralization against all four serotypes (complex-type, Table 3 ). On the other hand, five clones (one from acute-phase and four from convalescent-phase) of anti-NS1 HuMAbs were positive for viral neutralization activity against JEV, without viral neutralization activity against all four DV $(<50 \%$ against all four serotypes); whereas two clones (one from acute-phase and one from convalescent-phase) of anti-NS1 HuMAbs were positive for viral neutralization activity against JEV, with subcomplex-type viral neutralization activity against DV2, DV3, and DV4.

Figure 1 shows the staining of Vero cells infected with serotypes DV1 to DV4 and Vero cells infected with JEV by several HuMAb clones (anti-E, anti-prM, or anti-NS1, Figure 1A) and the percent reduction of viral replication in Vero cells incubated with the same HuMAb clones (Figure 1B).

In this study, hybridoma culture fluids were prepared under the same conditions. These culture fluids from individual hybridoma cells are also positive against DV by immunofluorescence. In addition, the same lots of individual culture fluids were used throughout the experiments in this study, such as immunofluorescence (against both DV and JEV), viral neutralization (against both DV and JEV), and western blotting test. The IgG concentration of the HuMAbs was not adjusted in this study. Therefore, there are some risks that some antibody may have low immunofluorescence, viral neutralization, or western blot signal, because it may be produced from hybridoma at a low concentration/amount.

\section{Cross-reactivity of HuMAbs with JEV as assessed by western blotting}

The cross-reactivity of HuMAbs with JEV antigens was also examined by western blotting. The results are summarized in Table 4. More than half of the anti-DV E HuMAbs were also reactive with JEV E on western blots. Also, most of the anti-DV NS1 HuMAbs were reactive with JEV NS1. In contrast, all of the anti-DV prM HuMAbs were not reactive with 
Table 3 Cross-reactivity of Japanese encephalitis virus with human monoclonal antibodies derived from dengue patients as assessed by the viral neutralization assay

\begin{tabular}{|c|c|c|c|c|c|c|c|c|c|}
\hline \multicolumn{4}{|c|}{ DV serotype ${ }^{a}$} & \multicolumn{6}{|c|}{ HuMAbs positive against DV in the $\mathrm{VN}$ assay (positive against JEV in the $\mathrm{VN}$ assay) } \\
\hline \multirow[t]{2}{*}{ DVI } & \multirow[t]{2}{*}{ DV2 } & \multirow[t]{2}{*}{ DV3 } & \multirow[t]{2}{*}{ DV4 } & \multicolumn{3}{|c|}{ Derived from acute-phase patients ${ }^{c}$} & \multicolumn{3}{|c|}{ Derived from convalescent-phase patients ${ }^{c}$} \\
\hline & & & & $\mathbf{E}$ & prM & NSI & E & prM & NSI \\
\hline$-\mathrm{d}$ & $-{ }^{\mathrm{d}}$ & $-{ }^{\mathrm{d}}$ & $-\mathrm{d}$ & $5(2 / 0)^{\mathrm{b}}$ & $5(0 / 0)$ & $3(1 / 0)$ & $\mathrm{I}(0 / 0)$ & $\mathrm{I}(0 / 0)$ & $6(1 / 3)$ \\
\hline+ & - & - & - & & I (0/0) & & & & \\
\hline- & + & - & - & $3(1 / 0)$ & & & & & \\
\hline- & - & + & - & $\mathrm{I}(0 / 0)$ & & & & & \\
\hline- & - & - & + & & $2(0 / 0)$ & & & & \\
\hline- & + & + & - & $2(0 / 0)$ & & & & & \\
\hline- & + & - & + & $\mathrm{I}(0 / 0)$ & & & & I $(0 / 0)$ & \\
\hline- & ++ & - & + & I $(0 / 0)$ & & & & & \\
\hline+ & + & + & - & $4(1 / 0)$ & & & & & \\
\hline+ & ++ & + & - & I $(I / 0)$ & & & & & \\
\hline+ & ++ & ++ & - & $\mathrm{I}(0 / 0)$ & & & & & \\
\hline+ & ++ & - & + & $\mathrm{I}(0 / 0)$ & & & & & \\
\hline- & + & + & + & $2(1 / 0)$ & & $\mathrm{I}^{\mathrm{e}}\left(\mathrm{I}^{\mathrm{e} / 0}\right)$ & & & $2^{f, g}\left(I^{f} / 0\right)$ \\
\hline- & ++ & + & + & $7(\mathrm{I} / \mathrm{I})$ & & & & & \\
\hline+ & + & + & + & $19(9 / 1)$ & & & & & \\
\hline+ & ++ & + & + & $17(7 / 0)$ & & & & & \\
\hline+ & + & + & ++ & $2(1 / 0)$ & & & & & \\
\hline++ & ++ & + & + & I $(0 / 0)$ & & & & & \\
\hline+ & ++ & ++ & + & $\mathrm{I}(0 / 0)$ & & & & & \\
\hline+ & ++ & + & ++ & II (5/0) & & & I (0/0) & & \\
\hline++ & ++ & + & ++ & $5(4 / 0)$ & & & & & \\
\hline+ & ++ & ++ & ++ & $3(2 / 1)$ & & & & & \\
\hline++ & ++ & ++ & ++ & II (8/0) & & & & & \\
\hline
\end{tabular}

Notes: ${ }^{a}-,<50 \% ;+, \geq 50 \%$ and $<90 \%$; and,$++ \geq 90 \%$ FFU reduction of DVI-DV4 replication compared with the negative control; ${ }^{26}$ bnumber of HuMAb clones reactive with JEV in the VN assay is shown in parentheses: number of clones showing $\geq 50$ and $<80 \%(+)$ FFU reduction of JEV replication compared with the negative control/number of clones showing $\geq 80 \%$ (++) FFU reduction of JEV replication compared with the negative control; 'viral proteins targeted by HuMAbs were identified using DV2 E, prM, and NSI proteins; ${ }^{26}$ dHuMAbs showing a positive reaction with DV by IF, but not in the VN assay ( $<50 \%$ reduction of the replication of any DV serotype); ${ }^{\circ} \mathrm{HuMAb} \mathrm{D} 30-2 \mathrm{BI} 55$ is also weakly reactive with DV2 E; 'HuMAb D25-2BIIGII is also weakly reactive with DV2 E and prM; sHuMAb D25-4D4FI0 is also weakly reactive with DV2 prM.

Abbreviations: DV, Dengue virus; E, envelope glycoprotein; HuMAb, human monoclonal antibody; prM, premembrane protein; NSI, nonstructural protein I; IF, immunofluorescence; VN, viral neutralization; FFU, focus-forming units; JEV, Japanese encephalitis virus.

JEV prM. Interestingly, three of the anti-DV E clones and two of the anti-DV NS1 clones that showed high neutralizing activity (++) against JEV showed no apparent reaction with $\mathrm{JEV}$ on the western blots.

\section{Discussion}

Overall, hybridomas previously generated from peripheral blood mononuclear cells isolated from dengue patients secondarily infected with $\mathrm{DV}^{26,27}$ were producers of HuMAbs cross-reactive with JEV-infected cells by immunofluorescence and showed high rates of neutralization against JEV by viral neutralization assay. The clones that cross-reacted with JEV and showed neutralizing activity against JEV were mainly classified into two groups: one contained clones that recognized DV E and were derived from acute-phase, and the other contained clones that recognized DV NS1 and were mostly derived from convalescent-phase. In contrast, none of the anti-DV prM clones showed neutralizing activity against JEV.

Of all the viruses that belong to the family Flaviviridae, JEV shows the closest antigenic relationship with West Nile virus, Murray Valley encephalitis virus, and St Louis encephalitis virus; JEV is much less antigenically related to DV. ${ }^{8,31}$ However, the present study showed that most of the anti-DV E antibodies from hybridomas generated from peripheral blood mononuclear cells from DV patients during the acute phase showed complex-type cross-reactions and neutralizing activity against all four DV serotypes; indeed, not only do these show strong viral neutralization activity against DV2 (which was replicating in these patients), they are also strongly crossreactive with other DV serotypes ${ }^{26}$ and with JEV. These results showed there are common antigenic sites between DV E and JEV E, although a low antigenic relationship exists between DV and JEV. This supports previous reports of flavivirus 
A

\section{D23-1C2D2 (anti-E)}
D23-1E12E8 (anti-E)

\section{D23-1D5G10 (anti-prM)}

D27-1E8A4 (anti-NS1)

D28-2B11F9 (anti-NS1)
DV1
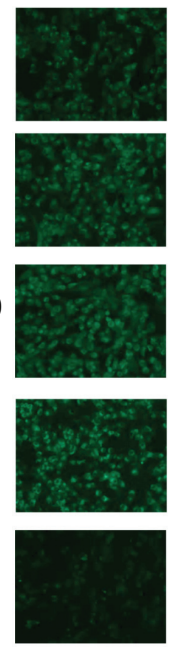

DV2
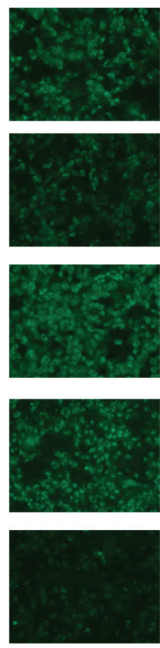

DV3
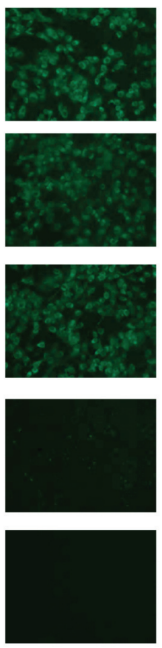

DV4
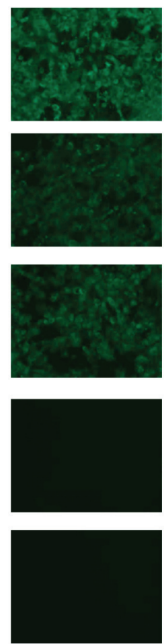

JEV
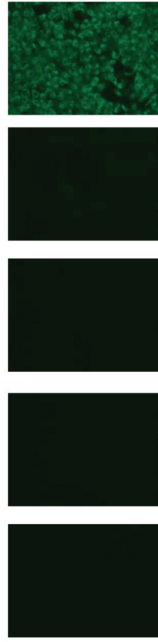

B

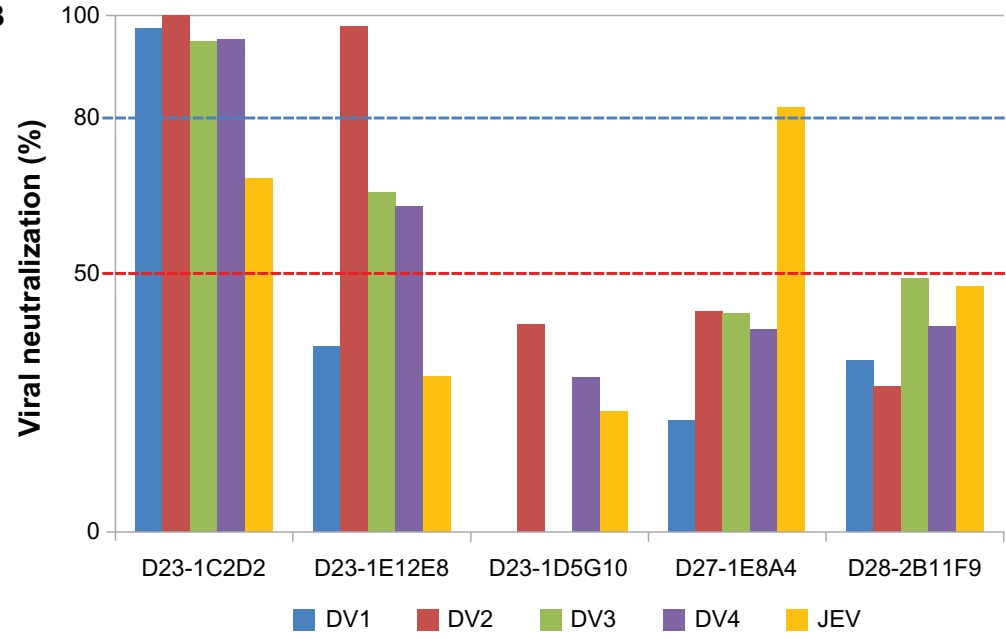

Figure I Staining of Vero cells infected with serotypes DVI to DV4 and of Vero cells infected with Japanese encephalitis virus using several human monoclonal antibody clones (anti-E, anti-prM, or anti-NSI). (A) Anti-DV E (D23-IC2D2 and D23-IEI2E8), anti-DV prM (D23-ID5GI0), and anti-DV NSI (D27-IE8A4 and D28-2BI IF9) clones were incubated with Vero cells infected with Dengue virus serotypes I-4 or with Vero cells infected with Japanese encephalitis virus. (B) Percent reduction of viral replication in Vero cells incubated with the same human monoclonal antibody clones.

Abbreviations: DV, Dengue virus; E, envelope glycoprotein; prM, premembrane protein; NSI, nonstructural protein I.

Table 4 Reactivity of dengue patient-derived human monoclonal antibodies with Japanese encephalitis virus-infected cell lysate on western blots

\begin{tabular}{|c|c|c|c|c|}
\hline \multirow[t]{2}{*}{$\begin{array}{l}\text { DV protein used as a target for } \\
\text { dengue patient-derived HuMAbs }\end{array}$} & \multirow[t]{2}{*}{$\begin{array}{l}\text { Reactivity with JEV-infected } \\
\text { cells on western blots }\end{array}$} & \multicolumn{3}{|c|}{$\begin{array}{l}\text { Dengue patient-derived HuMAb clone numbers } \\
\text { showing a positive activity against JEV in the } \\
\text { VN assay }\end{array}$} \\
\hline & & - & + & ++ \\
\hline \multirow[t]{2}{*}{$E$} & - & 22 & 17 & 3 \\
\hline & + & 33 & 26 & 0 \\
\hline \multirow[t]{2}{*}{$\mathrm{prM}$} & - & 10 & 0 & 0 \\
\hline & + & 0 & 0 & 0 \\
\hline \multirow[t]{2}{*}{ NSI } & - & 4 & 3 & 2 \\
\hline & + & I & I & I \\
\hline
\end{tabular}

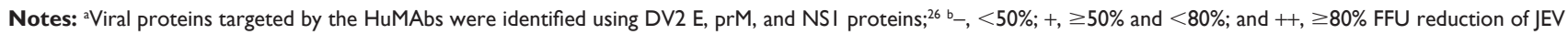
replication compared with that observed for the negative control.

Abbreviations: DV, Dengue virus; E, envelope glycoprotein; HuMAb, human monoclonal antibody; prM, premembrane protein; NSI, nonstructural protein I; VN, viral neutralization; JEV, Japanese encephalitis virus. 
envelope glycoprotein cross-reactive epitopes analyzed by mouse monoclonal antibodies indicating that DV and JEV envelope proteins have sequence similarity and also share structure similarity in causing the cross-activity effects. ${ }^{32,33}$ In addition, anti-DV NS1 antibodies showing neutralizing activity against DV are 3/4 clones from acute-phase and 2/8 clones from convalescent-phase, ${ }^{26}$ and $2 / 4$ clones from acutephase and 5/8 clones from convalescent-phase also showed neutralizing activity against JEV in the present study. There has been no report about cross-reactivity in flaviviruses. However, the NS1 gene shares a high degree of homology. ${ }^{34}$ The results of the present study also showed that anti-prM HuMAbs (eight derived from acute-phase patients and two from convalescent-phase patients) had no neutralizing activity against JEV. This supports the findings of a previous study showing that anti-prM antibodies in sera from DV-infected or JEV-infected patients during the convalescent phase did not react with either DV or JEV on western blots. ${ }^{35}$ The present study showed that most of the anti-DV E and anti-DV NS1 HuMAbs derived from dengue patients during the acute phase of a secondary infection (around one week after the onset of illness) neutralized JEV, but none of the anti-DV prM HuMAbs derived from acute-phase and convalescentphase patients neutralized JEV; this is despite the finding that anti-prM antibody responses are amplified after a secondary infection. ${ }^{36} \mathrm{~A}$ previous report described the generation of HuMAbs by transforming B cells derived from dengue patients (who were secondarily infected with DV) during the convalescent phase (15-24 days after defervescence). The results showed that $64 \%$ of anti-E, $7 \%$ of anti-NS1, and $3 \%$ of anti-prM antibodies were cross-reactive with JEV on

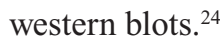

The E protein of flaviviruses is the principal antigen responsible for eliciting neutralizing antibody responses; however, neutralizing antibodies specific for the prM and NS1 proteins have also been detected. ${ }^{37-40}$ The anti-DV NS1 HuMAbs used in the present study were mostly derived from dengue patients during the convalescent phase of a secondary DV infection. ${ }^{26}$ Several anti-NS1 HuMAbs showed strong neutralizing activity against JEV, although those antibodies showed no apparent reactions with JEV-infected cells on immunofluorescence analysis (Table 1). A previous paper also reported several cases that were negative by immunofluorescence (anti-JEV IgG IIFT, Euroimmune, Lübeck, Germany), but positive by plaque reduction neutralization test. ${ }^{41}$ The present study examined three anti-NS1 clones that reacted with DV NS1, but also showed a weak crossreaction with DV E and/or prM. Two of the three showed neutralizing activity against JEV. A previous study reported that all convalescent serum samples obtained from Japanese encephalitis patients contained anti-JEV NS1 IgG antibodies. Of these, $65 \%$ and $40 \%$ contained JEV NS1-specific IgM and IgA antibodies, respectively; also, these IgM and IgA antibodies did not cross-react with $\mathrm{JEV}^{42}$ The anti-NS1 clones used in the present study (four clones obtained from acute-phase patients and eight from convalescent-phase patients) were all IgG antibodies. ${ }^{26}$

No information regarding the JEV infection or vaccination status of patients from whom the HuMAbs were derived was available; therefore, the possibility that the HuMAbs might have (at least in part) originated from memory immune cells that were initially primed with JEV antigens after a natural JEV infection or an anti-JEV vaccination cannot be ruled out. However, the acute phase of the disease in patients secondarily infected with heterotypic DV may be due to IgG antibodies induced by the secondary infection. Thus, it may be possible that some of the HuMAbs used in the present study could be derived from immune cells that had been primed after exposure to JEV.

\section{Acknowledgments}

The authors thank P Singhasivanon for valuable discussions, and P Sawanpanyalert and J Boon-Long for coordinating the project. This study was partly supported by Japan Science and Technology Agency/Japan International Cooperation Agency (SATREPS, 08080924), the Founding Research Center for Emerging and Re-emerging Infectious Diseases Program, which was launched through a project commissioned by the Ministry of Education, Culture, Sports, Science and Technology of Japan, and the Thailand Research Fund through the Royal Golden Jubilee PhD Program and Mahidol University (Grant PHD/0260/2550, to CP).

\section{Disclosure}

The authors report no conflicts of interest in this work.

\section{References}

1. Kuhn RJ, Zhang W, Rossmann MG, et al. Structure of dengue virus: implications for flavivirus organization, maturation, and fusion. Cell. 2002;108(5):717-725.

2. Innis BL, Thirawuth V, Hemachudha C. Identification of continuous epitopes of the envelope glycoprotein of dengue type 2 virus. Am J Trop Med Hyg. 1989;40(6):676-687.

3. Calisher CH, Karabatsos N, Dalrymple JM, et al. Antigenic relationships between flaviviruses as determined by cross-neutralization tests with polyclonal antisera. J Gen Virol. 1989;70 Pt 1:37-43.

4. Makino Y, Tadano M, Saito M, et al. Studies on serological crossreaction in sequential flavivirus infections. Microbiol Immunol. 1994;38(12):951-955. 
5. Chunsuttiwat S. Issues Related to Integration of JE Vaccine into National EPI: Experience from Thailand. Bangkok, Thailand: Department of Communicable Disease Control, Ministry of Public Health; 1998.

6. Martin DA, Biggerstaff BJ, Allen B, Johnson AJ, Lanciotti RS, Roehrig JT. Use of immunoglobulin M cross-reactions in differential diagnosis of human flaviviral encephalitis infections in the United States. Clin Diagn Lab Immunol. 2002;9(3):544-549.

7. Olsen SJ, Supawat K, Campbell AP, et al. Japanese encephalitis virus remains an important cause of encephalitis in Thailand. Int $J$ Infect Dis. 2010;14(10):e888-e892.

8. Mansfield KL, Horton DL, Johnson N, et al. Flavivirus-induced antibody cross-reactivity. J Gen Virol. 2011;92 Pt 12:2821-2829.

9. Grossman RA, Gould DJ, Smith TJ, Johnsen DO, Pantuwatana S. Study of Japanese encephalitis virus in Chiangmai Valley, Thailand. I. Introduction and study design. Am J Epidemiol. 1973;98(2):111-120.

10. van der Schaar HM, Wilschut JC, Smit JM. Role of antibodies in controlling dengue virus infection. Immunobiology. 2009;214(7):613-629.

11. Halstead SB, O'Rourke EJ. Dengue viruses and mononuclear hagocytes. I. Infection enhancement by non-neutralizing antibody. J Exp Med. 1977;146(1):201-217.

12. Sangkawibha N, Rojanasuphot $S$, Ahandrik S, et al. Risk factors in dengue shock syndrome: a prospective epidemiologic study in Rayong, Thailand. I. The 1980 outbreak. Am J Epidemiol. 1980;120(5): 653-669.

13. Reiter P. Yellow fever and dengue: a threat to Europe? Euro Surveill. 2010;15(10):19509.

14. García G, Sierra B, Pérez AB, et al. Asymptomatic dengue infection in a Cuban population confirms the protective role of the RR variant of the FcgammaRIIa polymorphism. Am J Trop Med Hyg. 2010;82(6): 1153-1156.

15. Harris E, Videa E, Pérez L, et al. Clinical, epidemiologic, and virologic features of dengue in the 1998 epidemic in Nicaragua. Am J Trop Med Hyg. 2000;63(1-2):5-11.

16. Sabin AB. Research on dengue during World War II. Am J Trop Med Hyg. 1952;1(1):30-50.

17. Halstead SB, Chow J, Marchette NJ. Immunological enhancement of dengue virus replication. Nat New Biol. 1973;243(122):24-26.

18. Hoke CH, Nisalak A, Sangawhipa N, et al. Protection against Japanese encephalitis by inactivated vaccines. $N$ Engl J Med. 1988;319(10): 608-614.

19. Gibbons RV, Kalanarooj S, Jarman RG, et al. Analysis of repeat hospital admissions for dengue to estimate the frequency of third or fourth dengue infections resulting in admissions and dengue hemorrhagic fever, and serotype sequences. Am J Trop Med Hyg. 2007;77(5):910-913.

20. Anderson KB, Gibbons RV, Thomas SJ, et al. Pre-existing Japanese encephalitis virus neutralizing antibodies and increased symptomatic dengue illness in a school-based cohort in Thailand. PLoS Negl Trop Dis. 2011;5(10):e1311.

21. Wahala WM, Kraus AA, Haymore LB, Accavitti-Loper MA, de Silva AM. Dengue virus neutralization by human immune sera: role of envelope protein domain III-reactive antibody. Virology. 2009; 392(1):103-113.

22. Beltramello M, Williams KL, Simmons CP, et al. The human immune response to dengue virus is dominated by highly cross-reactive antibodies endowed with neutralizing and enhancing activity. Cell Host Microbe. 2010;8(3):271-283.

23. Schieffelin JS, Costin JM, Nicholson CO, et al. Neutralizing and nonneutralizing monoclonal antibodies against dengue virus $\mathrm{E}$ protein derived from a naturally infected patient. Virol J. 2010;7:28.

24. Dejnirattisai W, Jumnainsong A, Onsirisakul N, et al. Cross-reacting antibodies enhance dengue virus infection in humans. Science. 2010;328(5979):745-748.

25. de Alwis R, Beltramello M, Messer WB, et al. In-depth analysis of the antibody response of individuals exposed to primary dengue virus infection. PLoS Negl Trop Dis. 2011;5(6):e1188.
26. Setthapramote C, Sasaki T, Puiprom O, et al. Human monoclonal antibodies to neutralize all dengue virus serotypes using lymphocytes from patients at acute phase of the secondary infection. Biochem Biophys Res Commun. 2012;423(4):867-872.

27. Sasaki T, Setthapramote C, Kurosu T, et al. Dengue virus neutralization and antibody-dependent enhancement activities of human monoclonal antibodies derived from dengue patients at acute phase of secondary infection. Antiviral Res. 2013;98(3):423-431.

28. Kurosu T, Khamlert C, Phanthanawiboon S, Ikuta K, Anantapreecha S. Highly efficient rescue of dengue virus using a co-culture system with mosquito/mammalian cells. Biochem Biophys Res Commun. 2010;394(2):398-404.

29. Okuno Y, Igarashi A, Fukai K. Neutralization tests for dengue and Japanese encephalitis viruses by the focus reduction method using peroxidase-anti-peroxidase staining. Biken J. 1978;21(4):137-147.

30. Falconar AK. Identification of an epitope on the dengue virus membrane $(\mathrm{M})$ protein defined by cross-protective monoclonal antibodies: design of an improved epitope sequence based on common determinants present in both envelope (E and M) proteins. Arch Virol. 1999;144(12):2313-2330.

31. Kimura-Kuroda J, Yasui K. Antigenic comparison of envelope protein E between Japanese encephalitis virus and some other flaviviruses using monoclonal antibodies. J Gen Virol. 1986;67 Pt 12:2663-2672.

32. Heinz FX, Stiasny K. Flaviviruses and their antigenic structure. J Clin Virol. 2012;5(4):289-295.

33. Crill WD, Chang GJ. Localization and characterization of flavivirus envelope glycoprotein cross-reactive epitopes. J Virol. 2004;78(24): 13975-13986.

34. Muller DA, Young PR. The flavivirus NS1 protein: molecular and structural biology, immunology, role in pathogenesis and application as a diagnostic biomarker. Antiviral Res. 2013;98(2):192-208.

35. Cardosa MJ, Wang SM, Sum MSH, Tio PH. Antibodies against prM protein distinguish between previous infection with dengue and Japanese encephalitis viruses. BMC Microbiol. 2002;2:9.

36. Lai CY, Tsai WY, Lin SR, et al. Antibodies to envelope glycoprotein of dengue virus during the natural course of infection are predominantly cross-reactive and recognize epitopes containing highly conserved residues at the fusion loop of domain II. $J$ Virol. 2008;82(13): 6631-6643.

37. Pincus S, Mason PW, Konishi E, et al. Recombinant vaccinia virus producing the prM and $\mathrm{E}$ proteins of yellow fever virus protects mice from lethal yellow fever encephalitis. Virology. 1992;187(1): 290-297.

38. Colombage G, Hall R, Pavy M, Lobigs M. DNA-based and alphavirusvectored immunisation with prM and E proteins elicits long-lived and protective immunity against the flavivirus, Murray Valley encephalitis virus. Virology. 1998;250(1):151-163.

39. Shu PY, Chen LK, Chang SF, et al. Dengue NS1-specific antibody responses: isotype distribution and serotyping in patients with dengue fever and dengue hemorrhagic fever. J Med Virol. 2000;62(2): 224-232.

40. Chung KM, Thompson BS, Fremont DH, Diamond MS. Antibody recognition of cell surface-associated NS1 triggers Fc-gamma receptormediated phagocytosis and clearance of West Nile virus-infected cells. J Virol. 2007;81(17):9551-9555.

41. Litzba N, Klade CS, Lederer S, Niedrig M. Evaluation of serological diagnostic test systems assessing the immune response to Japanese encephalitis vaccination. PLoS Negl Trop Dis. 2010;4(11):e883.

42. Shu PY, Chen LK, Chang SF, et al. Antibody to the nonstructural protein NS1 of Japanese encephalitis virus: potential application of mAb-based indirect ELISA to differentiate infection from vaccination. Vaccine. 2001;19(13-14):1753-1763. 


\section{Supplementary material}

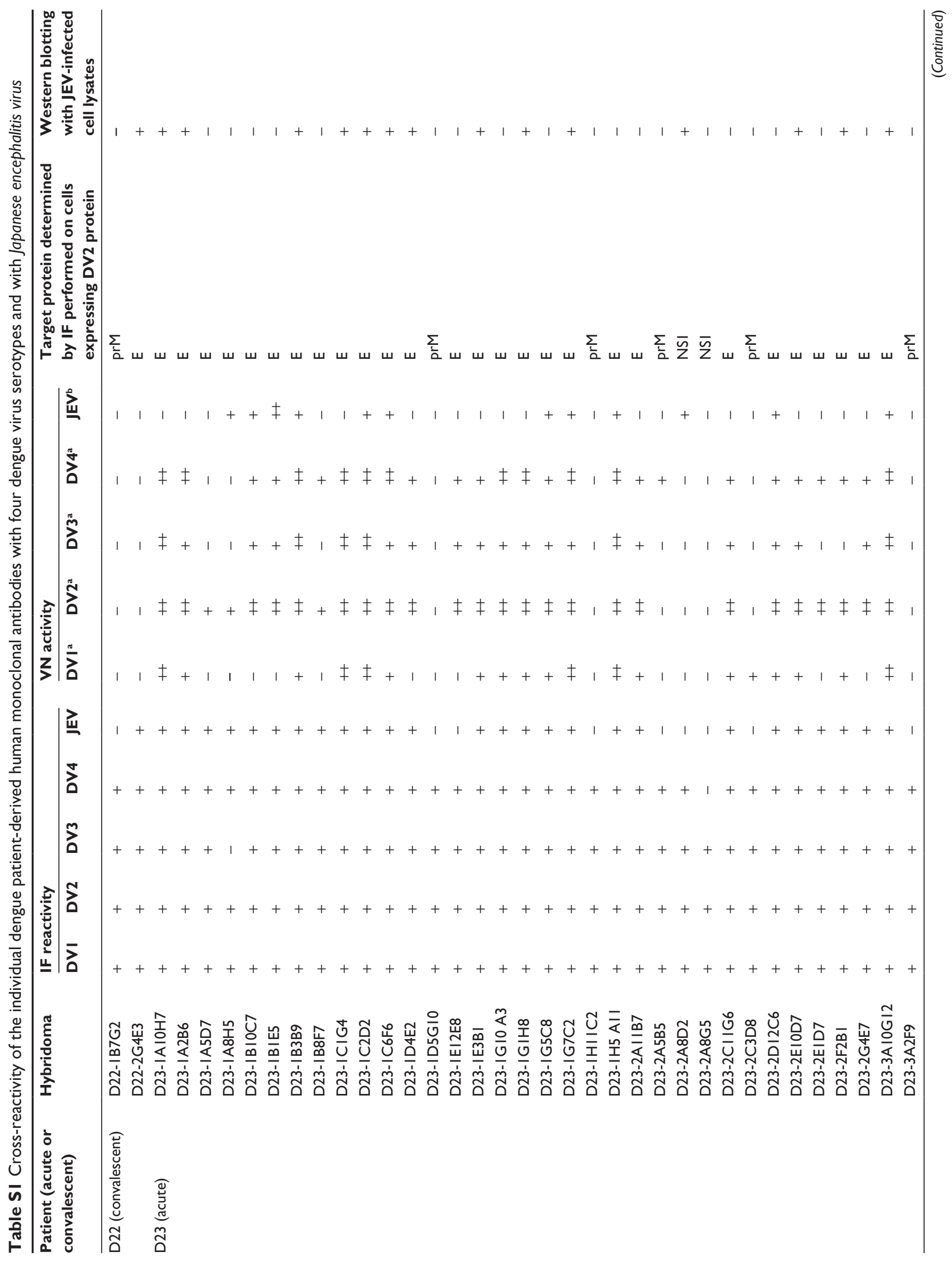




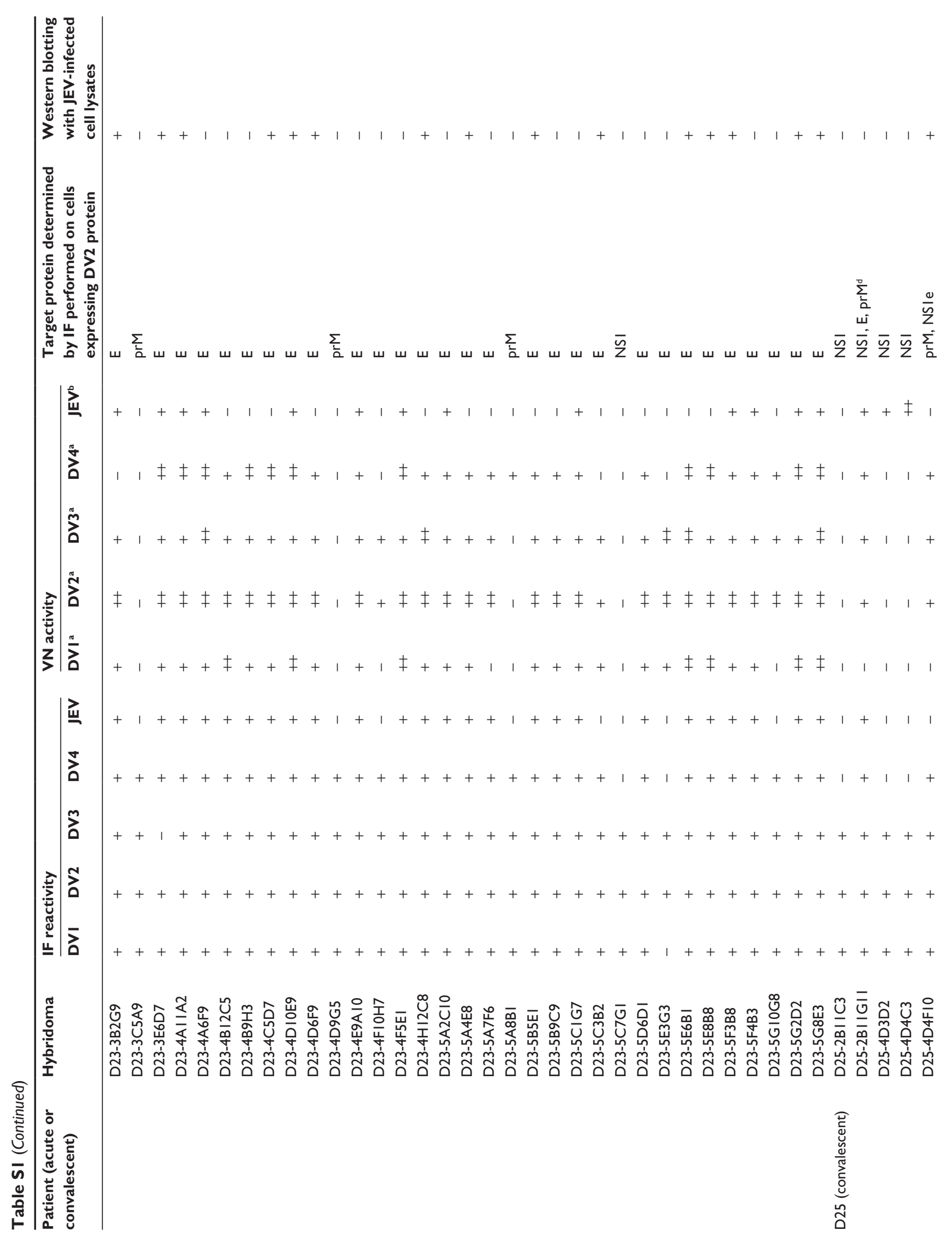




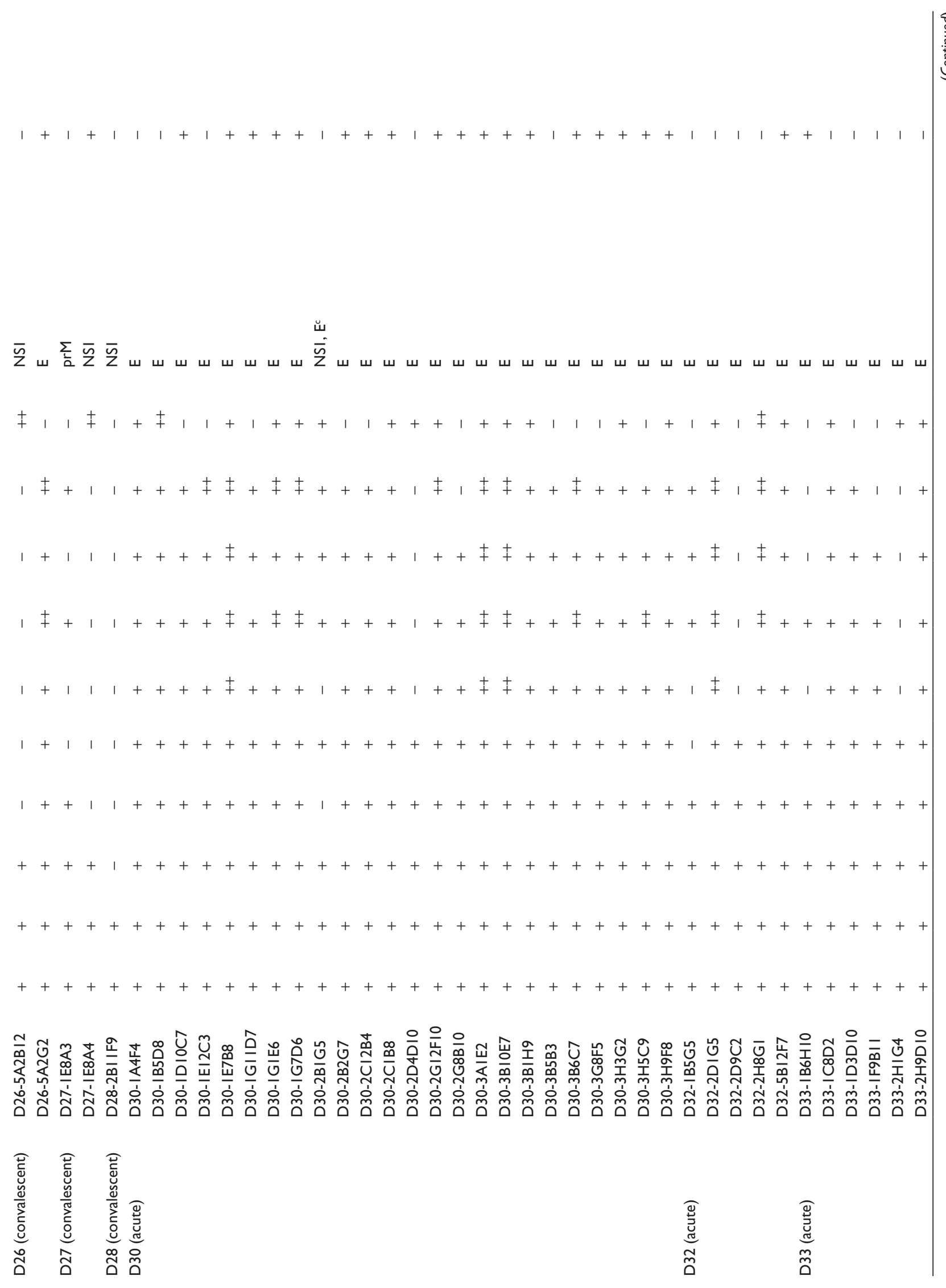




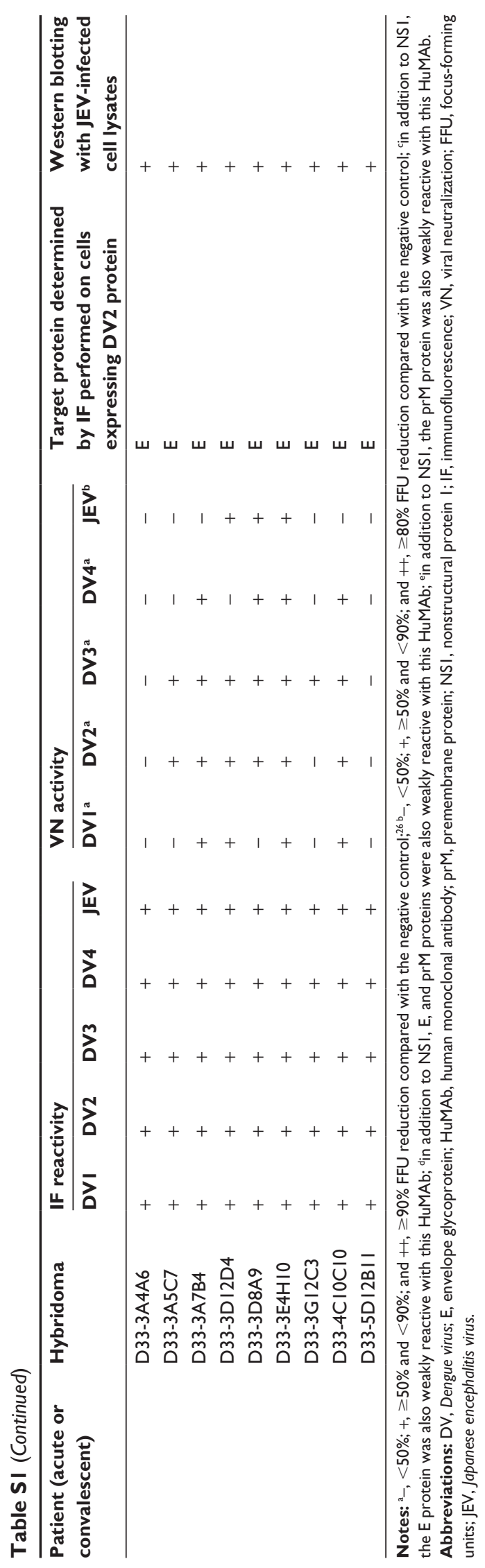


Biologics: Targets \& Therapy

\section{Publish your work in this journal}

Biologics: Targets \& Therapy is an international, peer-reviewed journal focusing on the patho-physiological rationale for and clinical application of Biologic agents in the management of autoimmune diseases, cancers or other pathologies where a molecular target can be identified. This journal is indexed on PubMed Central, CAS, EMBase, Scopus

Submit your manuscript here: http://www.dovepress.com/biologics-targets--therapy-journal

and the Elsevier Bibliographic databases. The manuscript management system is completely online and includes a very quick and fair peerreview system, which is all easy to use. Visit http://www.dovepress. com/testimonials.php to read real quotes from published authors. 